\title{
Multipath-channel estimation and application to ionospheric channels
}

\author{
José L. Sanz-González • Santiago Zazo • \\ Iván A. Pérez-Álvarez · Javier López-Pérez
}

Published online: 12 June 2011

(C) Springer Science+Business Media, LLC 2011

\begin{abstract}
In this paper, the theory of linear time-variant systems is applied to multipath channels and, in particular, to ionospheric channels. Some useful formulas in time and frequency domains are given for both deterministic and random linear time-variant systems and multipath channels. The fundamental parameters of a multipath channel are the delay and the gain coefficient of each path, and the number of significant paths. We propose a methodology for estimating path-delays and path-coefficients and its application to the ionospheric channel estimation. Also, if path coefficients are stationary random processes, the power spectral density of the coefficients can be estimated from measured data of real (actual) channels. Finally, exhaustive computer simulations have been realized for testing the algorithms and a sample of the results is provided in the paper; also, estimation results for an actual narrowband HF ionospheric channel are provided, where up to four significant paths (rays) are detected with a Doppler spread lower than $0.2 \mathrm{~Hz}$.
\end{abstract}

\footnotetext{
J. L. Sanz-González (凶) · S. Zazo

Dpto. SSR-ETSI de Telecomunicación-UPM, Universidad

Politécnica de Madrid (UPM), Ciudad Universitaria,

28040 Madrid, Spain

e-mail: jlsanz@gcs.ssr.upm.es

S. Zazo

e-mail: santiago@gaps.ssr.upm.es

I. A. Pérez-Álvarez · J. López-Pérez

Dpto. Señales y Comunicaciones, Universidad de Las Palmas de

Gran Canaria (ULPGC), IDeTIC, Campus de Tafira,

35017 Las Palmas, Spain

e-mail: iperez@dsc.ulpgc.es

J. López-Pérez

e-mail: jlopez@cetic.eu
}

Keywords Linear time-variant systems - Multipath channels · Ionospheric channels $\cdot$ Path delay $\cdot$ Doppler spread · Fading channels $\cdot$ Gaussian spectrum

\section{Introduction}

The characterization of linear time-variant channels has been done more than 50 years ago [1-3]. Zadeh [1] established the frequency analysis of "linear variable networks" (linear time-variant systems) by defining transfer functions (depending on frequency and time) as a generalization of transfer functions of "linear fixed networks" (linear time-invariant systems). Kailath [2] studied the statistical characterization of random channels using the two-dimensional impulse response of time-variant systems. Bello [3] defined different input-output relations in time and frequency domains applied to linear time-variant systems, conveying different types of transfer functions; also, he extended this study to random channels by defining the corresponding correlation functions of those transfer functions. In [4], Bello extended the tapped delay line model of narrowband channels presented in [3] to ultrawideband mobile channels.

Watterson et al. [5] analyzed high frequency (HF) ionospheric channels from measured data, and they simulated those channels by transversal filters (tapped delay lines) with time-variant coefficients, concluding that for narrowband channels (up to $12 \mathrm{kHz}$ ) the hypotheses about the Gaussian characterization of the coefficients (Gaussian processes and Gaussian spectra) are fulfilled. Also, in [5], a physical interpretation of the results is given in terms of ionospheric layers and magnetoionic components. In [6], a new simple narrowband HF ionospheric channel model is introduced by modeling independently delay and Doppler 
effects. On the other hand, models for wideband (up to $1 \mathrm{MHz}) \mathrm{HF}$ ionospheric channels are presented in [7, 8].

As is well known, a radio HF link using ionospheric reflections is an important alternative to satellite link when cost, confidence and security of communications are considered. However, the HF link implies a dramatic distortion that has to be compensated by means of sophisticated communication systems. Although HF communications decreased sharply in the civil field after the second half of the twentieth century, HF communications systems remained in use for military applications due to the technological challenge for strategic communications, and as an attractive alternative to other radio communications. After some years, most of this technology has been unclassified, being allowed civil applications. Presently, there is a large expertise in this field for both civil and military applications; for example, (a) strategic communications related to data transmission or coded voice between static stations and/or mobile terminals, (b) multi-user communications between vessels or terrestrial vehicles (dealing with no lineof-sight in long-distance links) as an alternative or back-up system to the satellite links, (c) point-to-point communications for establishing a specific data or voice link as an alternative to a rented satellite link.

In this paper, we consider a general multipath channel model, where path delays and path-coefficients are timedependent (model useful for both narrow- and wide- band channels). The challenge here is the reliable estimations of the path parameters from measured data. In fact, we have a matrix of data (two-dimensional data) that corresponds to frequency and time, as it will be seen later. Then, the problem is reduced to the estimation of sinusoidal signals (amplitudes, phases and frequencies). This is a classical problem in spectral analysis [9, chap. 16], but the wellknown Prony's method does not work adequately under noisy data [9, pp. 1193], and other methods based on eigen-structures (based on estimations of the correlation matrix) are not applied here due to the non-stationary character of the problem. Consequently, we propose a new methodology for this estimation problem to cope with noisy data and non-stationary signals. Our methodology estimates path delays at a first stage, and, then, estimates path coefficients after incorporating delay estimations. Algorithms for solving this estimation problem have been developed by the authors of this paper and some results will be shown later. Note that once the parameters of a real ionospheric channel are estimated, one can implement (simulate) a controllable model of the real channel in order to test communication systems or to improve the design of systems to be used over those actual channels. Note that the proposed estimation methodology is valid for any multipath channel and, in particular, for ionospheric channels.
The paper is organized as follows. In Sect. 2, we start with the idea of time convolution integral in a linear time-variant system, conveying to a frequency convolution integral (see Appendix 1); also, we provide some new interesting formulas for the determination of the output power spectral density (see Appendix 2). In Sect. 3, we present a methodology for estimating the parameters of a multipath channel by estimating the path delays and path-coefficients (applying to noisy data and non-stationary signals). Section 4 shows estimation results for modeled channels and, Sect. 5, for actual ionospheric channels. Finally, Sect. 6 summarizes the main conclusions of the paper.

\section{Multipath channel model}

Any linear time-variant system can be characterized by the convolution integral as follows $[1,10,11]$

$y(t)=\int_{-\infty}^{\infty} h(\tau ; t) x(t-\tau) d \tau$,

where $x(t)$ is the input signal, $y(t)$ is the output signal and $h(\tau ; t)$ is the unit-impulse response of the time-variant system, i.e. the response of the system at time $t$ to a unit impulse (a Dirac delta function) located at time $t-\tau$. If $h(\tau ; t)$ does not depend on $t$, we have the well-known linear time-invariant system. In Appendices 1 and 2, we develop some formulas in time and frequency domains applied to time-variant systems and related to the content of this paper. In the sequel, we are working with the equivalent low-pass complex-envelope signals.

According to [4], the impulse response $h(\tau ; t)$ of a general multipath channel may be modeled by

$h(\tau ; t)=\sum_{k=1}^{K} a_{k}(t) \cdot g\left(\tau-\tau_{k}(t)\right)$,

where $K$ is the number of paths between the transmitter and the receiver, $a_{k}(t)$ is the complex coefficient of the $k t h$-path and $\tau_{k}(t)$ is the corresponding path delay, $g(t)$ is the equivalent impulse response of the transmitter filters. (The effect of the receiver filters will be included later).

Now, taking Eqs. 2 into 29 of Appendix 1, we obtain the transfer function $H(j \omega ; t)$ of the multipath channel

$H(j \omega ; t)=\sum_{k=1}^{K} a_{k}(t) e^{-j \omega \cdot \tau_{k}(t)} \cdot G(j \omega)$,

where $G(j \omega)$ is the equivalent transfer function of the transmitter filters. If $\tau_{k}(t)$ is independent of time $t$, from Eq. 34 of Appendix 1 with $v_{k}=0$, we have

$Y(j \Omega)=\frac{1}{2 \pi} \sum_{k=1}^{K} A_{k}(j \Omega) *\left[G(j \Omega) X(j \Omega) e^{-j \Omega \cdot \tau_{k}}\right]$, 
where symbol $*$ means convolution operation, $X(j \Omega)$ and $Y(j \Omega)$ are the Fourier transforms of the input signal $x(t)$ and the output signal $y(t)$, respectively. The path-input spectrum $G(j \Omega) X(j \Omega)$ suffers a linear-phase change and a spectral spread due to the convolution by the spectrum $A_{k}(j \Omega)$ of each coefficient $a_{k}(t)$. The effects of the receiver filters $R(j \Omega$ ) should be consider now, and it is supposed that the passband of the receiver is wide enough so that $R(j \Omega) \cdot Y(j \Omega) \approx Y(j \Omega)$; furthermore, the additive noise of the channel is filtered by the receiver filters $R(j \Omega)$.

For stationary multipath channels, the autocorrelation function of $h(\tau ; t)$ defined in Eq. 2, can be obtained from Appendix 2 (see Eq. 38), and it is given by

$r_{h}(\Delta \tau ; \Delta t)=\sum_{k=1}^{K} \sum_{l=1}^{K} E\left\{a_{k}^{*}(t) a_{l}(t+\Delta t)\right\} \cdot r_{g}\left(\Delta \tau+\tau_{k}-\tau_{l}\right)$,

where $a_{k}^{*}(t)$ is the complex conjugate of $a_{k}(t)$. In Eq. 5 , it is supposed that $\tau_{k}$ does not depend on $t$, and that $r_{g}(\Delta \tau)$ is the autocorrelation function of the impulse response $g(\tau)$ of the transmitter filter, i.e.

$r_{g}(\Delta \tau)=\int_{-\infty}^{\infty} g^{*}(\tau) g(\tau+\Delta \tau) d \tau$

Supposing that path $k t h$ is independent of path $l t h$, Eq. 5 can be written as

$r_{h}(\Delta \tau ; \Delta t)=\sum_{k=1}^{K} r_{a_{k}}(\Delta t) \cdot r_{g}(\Delta \tau)$,

where $r_{a_{k}}(\Delta t)$ is the autocorrelation function of the path coefficient $a_{k}(t)$, supposed stationary. Now, taken Eqs. 7 into 40 of Appendix 2, we have

$S_{h}(j \omega ; j \Omega)=\sum_{k=1}^{K} S_{a_{k}}(j \Omega) \cdot|G(j \omega)|^{2}$,

where $S_{a_{k}}(j \Omega)$ is the power spectrum of path coefficient $a_{k}(t), G(j \omega)$ is the transfer function of the transmitter filters and $S_{h}(j \omega ; j \Omega)$ is the two-dimensional Fourier transform of $r_{h}(\Delta \tau ; \Delta t)$.

Finally, taken Eq. 8 into 39 of Appendix 2, we have

$S_{y}(j \Omega)=\frac{1}{2 \pi} \sum_{k=1}^{K}\left[|G(j \Omega)|^{2} S_{x}(j \Omega)\right] * S_{a_{k}}(j \Omega)$,

where $S_{x}(j \Omega)$ and $S_{y}(j \Omega)$ are the power spectral densities of $x(t)$ and $y(t)$, respectively. Formula Eq. 9 indicates that the path-input power spectrum is widened at the output due to the convolution with the power spectrum of each path coefficient. Therefore, the computation of coefficient power spectra is highly important in order to establish the degree of distortion over the transmitted signals: the wider coefficient spectra, the larger distortion in transmitted signals.

\section{New approach for multipath parameter estimation: application to the ionospheric channel}

Suppose we consider a multipath channel, defined by Eq. 2 or by the equivalent Eq. 3. Our purpose is to estimate complex coefficients $a_{k}(t)$ and delays $\tau_{k}(t)$ from noisy measurements of $H(j \omega ; t)$; then, $H\left(j \omega_{m} ; t_{n}\right), m=1,2, \ldots$, $M$, and $n=1,2, \ldots, N$, should represent $H(j \omega ; t)$. Also, it is supposed that $G\left(j \omega_{m}\right) \approx 1$ for $m=1,2, \ldots, M$, i.e. the frequency samples are inside the transmitter passband. (If $G(j \omega) \neq 1$, we divide both sides of Eq. 3 by $G(j \omega))$.

Now, the estimation problem can be established as follows: Given a collection of noisy values $H\left(j \omega_{m} ; t_{n}\right)$; $m=1,2, \ldots, M ; n=1,2, \ldots, N$, find the best approximation to coefficients $a_{k}\left(t_{n}\right)$ and delays $\tau_{k}\left(t_{n}\right), k=1,2, \ldots, K$, according to the following structure

$$
\begin{aligned}
H\left(j \omega_{m} ; t_{n}\right) & \approx \sum_{k=1}^{K} a_{k}\left(t_{n}\right) e^{-j \omega_{m} \cdot \tau_{k}\left(t_{n}\right)} ; m=1,2, \ldots, M ; n \\
& =1,2, \ldots, N
\end{aligned}
$$

where $\omega_{m}, t_{n}, M$ and $N$ are known beforehand, $a_{k}$ is a complex parameter, $\tau_{k}$ is a real parameter and $K$ is an integer.

A good criterion to solve this problem is the minimization of the quadratic error, defined by

$\sum_{n=1}^{N} \varepsilon_{n}\left(\mathbf{a}_{n}, \tau_{n}\right)=\sum_{n=1}^{N} \sum_{m=1}^{M}\left|H\left(j \omega_{m} ; t_{n}\right)-\sum_{k=1}^{K} a_{k}\left(t_{n}\right) e^{-j \omega_{m} \cdot \tau_{k}\left(t_{n}\right)}\right|^{2}$,

where $\mathbf{a}_{n}=\left[a_{1}\left(t_{n}\right), a_{2}\left(t_{n}\right), \ldots, a_{K}\left(t_{n}\right)\right]^{T}$ and $\tau_{n}=\left[\tau_{1}\left(t_{n}\right)\right.$, $\left.\tau_{2}\left(t_{n}\right), \ldots, \tau_{K}\left(t_{n}\right)\right]^{T}$ are column vectors.

Note that the minimization of the total error is equivalent to the minimization of each isolated error $\varepsilon_{n}\left(\mathbf{a}_{n}, \tau_{n}\right)$, $n=1,2, \ldots, N$, defined in Eq. 11, because these errors are independent of each other. In order to simplify the notation in the process of minimizing $\varepsilon_{n}\left(\mathbf{a}_{n}, \tau_{n}\right)$, we will discard the reference to time $t_{n}$ so that it can be written as

$\varepsilon(\mathbf{a}, \tau)=\sum_{m=1}^{M}\left|e_{m}(\mathbf{a}, \tau)\right|^{2}=\sum_{m=1}^{M} e_{m}^{*}(\mathbf{a}, \tau) \cdot e_{m}(\mathbf{a}, \tau)$

where

$e_{m}(\mathbf{a}, \tau)=H\left(j \omega_{m} ; t_{n}\right)-\sum_{k=1}^{K} a_{k} e^{-j \omega_{m} \cdot \tau_{k}}$,

being $\mathbf{a}=\left[a_{1}, a_{2}, \ldots, a_{K}\right]^{T}$ and $\tau=\left[\tau_{1}, \tau_{2}, \ldots, \tau_{K}\right]^{T}$, in fact $a_{k}=a_{k}\left(t_{n}\right), \tau_{k}=\tau_{k}\left(t_{n}\right), k=1,2, \ldots, K$. 
Equation 12 has a local or global minimum (or maximum) at $\mathbf{a}$ and $\tau$ if

$\sum_{m=1}^{M}\left(\frac{\partial e_{m}(\mathbf{a}, \tau)}{\partial a_{k}}\right)^{*} \cdot e_{m}(\mathbf{a}, \tau)=0, \quad k=1,2, \ldots, K$,

$\mathrm{R}_{e}\left\{\sum_{m=1}^{M}\left(\frac{\partial e_{m}(\mathbf{a}, \tau)}{\partial \tau_{k}}\right)^{*} \cdot e_{m}(\mathbf{a}, \tau)\right\}=0, \quad k=1,2, \ldots, K$,

where $\mathrm{R}_{\mathrm{e}}\{x\}$ means real part of $x$.

After some manipulations, Eqs. 14 and 15 can be expressed in matrix notation as follows

$\mathbf{M}_{e}^{*} \cdot\left(\mathbf{h}-\mathbf{M}_{e} \cdot \mathbf{a}\right)=\mathbf{0}$,

$\mathrm{I}_{m}\left\{\left(\mathbf{M}_{e} \cdot \operatorname{diag}(\mathbf{a})\right)^{*} \cdot \operatorname{diag}(\omega) \cdot\left(\mathbf{h}-\mathbf{M}_{e} \cdot \mathbf{a}\right)\right\}=\mathbf{0}$,

where ${ }^{*}$ stands for complex-conjugate transpose, $\mathrm{I}_{m}\{x\}$ means imaginary part of $x$, and

$\mathbf{M}_{e}=\left[\begin{array}{llll}e^{-j \omega_{1} \tau_{1}} & e^{-j \omega_{1} \tau_{2}} & \cdots & e^{-j \omega_{1} \tau_{K}} \\ e^{-j \omega_{2} \tau_{1}} & e^{-j \omega_{2} \tau_{2}} & \cdots & e^{-j \omega_{2} \tau_{K}} \\ \vdots & \vdots & \cdots & \vdots \\ e^{-j \omega_{M} \tau_{1}} & e^{-j \omega_{M} \tau_{2}} & \cdots & e^{-j \omega_{M} \tau_{K}}\end{array}\right]$

vector $\mathbf{h}=\left[h_{1}, h_{2}, \ldots, h_{M}\right]^{T}$, , where $h_{m}=H\left(j \omega_{m} ; t_{n}\right)$, and $t_{n}$ is fixed in the minimization process; vectors $\mathbf{a}=\left[a_{1}\right.$, $\left.a_{2}, \ldots, a_{K}\right]^{T}, \tau=\left[\tau_{1}, \tau_{2}, \ldots, \tau_{K}\right]^{T}$ are defined above, and $\boldsymbol{\omega}=\left[\omega_{1}, \omega_{2}, \ldots, \omega_{K}\right]^{T}$. Finally, $\operatorname{diag}(\mathbf{v})$ is a diagonal matrix with the components of vector $\mathbf{v}$ in the main diagonal.

Equations 16 and 17 are nonlinear in vectors $\mathbf{a}$ and $\tau$, consequently we cannot solve them analytically. On the other hand, any standard (gradient, genetic, etc.) minimization algorithm applied to Eqs. 12 and 13 does not work adequately because of the large amount of local minima. Also, the global minimum of Eq. 12 is very critical and sensitive with respect to vector $\tau$. In the following, we present a new algorithm for solving this estimation problem.

We describe the algorithm useful for estimating vectors $\tau$ and a that minimize Eq. 12. First, note that if vector $\tau$ is known, vector a can be obtained by solving Eq. 16, i.e.

$\mathbf{a}=\left(\mathbf{M}_{e}^{*} \cdot \mathbf{M}_{e}\right)^{-1} \cdot \mathbf{M}_{e}^{*} \cdot \mathbf{h}$

Therefore, the problem is to estimate the delay vector $\tau$, and the solution will be done iteratively.

\subsection{First estimation of path delays}

The idea for estimating the delays $\tau=\left[\tau_{1}\left(t_{n}\right)\right.$, $\left.\tau_{2}\left(t_{n}\right), \ldots, \tau_{K}\left(t_{n}\right)\right]^{T}$ is as follows: suppose we know $H\left(j \omega ; t_{n}\right)$ in the passband of the measurement system, and that $W_{B}(j \omega)$ is a frequency window narrower than the system passband; now, take the inverse Fourier transform of $H\left(j \omega ; t_{n}\right) \cdot W_{B}(j \omega)$ and consider the modulus of the result (in the $\tau$-domain), i.e. $\left|h\left(\tau ; t_{n}\right) * w_{B}(\tau)\right|=\left|\sum_{k=1}^{K} a_{k}\left(t_{n}\right) \cdot w_{B}\left(\tau-\tau_{k}\left(t_{n}\right)\right)\right|$,

where $|z|$ means "magnitude of $z$ ", $w_{B}(\tau)$ is the inverse Fourier transform of $W_{B}(j \omega)$, and $*$ means convolution operation. Therefore, to detect adequately $\tau_{k}$ by a peak detector, the separation among delays $\tau_{k}, k=1,2, \ldots, K$, must be greater than the time window length $w_{B}(\tau)$ which is the inverse value of the frequency window length $W_{B}(j \omega)$; also, the side-lobe aliasing and the noise of the measured data perturb the estimation of $\tau_{k}$. In fact, for separations among path-delays greater than the length of the time window $w_{B}(\tau)$, the peak detector over Eq. 20 is a quasi-optimum detector under white Gaussian noise, because the referred approach is related to the matched filter applied to each $k t h$-ray $(k=1,2, \ldots, K)$. It is important to use an adequate frequency window $W_{B}(j \omega)$ for minimizing time-aliasing and noise effects on expression Eq. 20. In our application, we have used a Kaiser window for $W_{B}(j \omega)$ with a shape parameter $\beta=2$ (see, for example, [9]).

In order to estimate $\tau_{k}$ in Eq. 20 at time $t_{n}$, we use a threshold for detecting possible intervals of $\tau$-values that contain several close (neighboring) $\tau_{k}$ 's. The threshold should be estimated from the noise level, and set adequately in order to prevent noise-peak detections. If the length of a detected interval is lower than the window length of $w_{B}(\tau)$, it is supposed that there is one $\tau_{k}$ in the middle of the referred interval. If the length of the detected interval is greater than a $w_{B}(\tau)$ window length, and lower than two delay-window lengths, then it is supposed that there are two $\tau_{k}$ 's out of $K$ in the referred interval, and so on. Therefore, with this method, multiple $\tau_{k}$ 's can be estimated inside a detected peak-interval, depending on the interval length, resulting in a delay resolution lower than a half of the delay-window length $w_{B}(\tau)$.

Furthermore, for very close paths, it is difficult to detect the paths with low intensity (say, lower than a tenth of the largest intensity). For these cases, we have proposed a procedure to cope with this problem of ray masking, which consists on to remove all the strongest rays after they have been estimated (i.e. subtracting them from the data), and then to estimate successively the lower rays. Consequently, our algorithm incorporates a first estimation of the strongest rays: some $\tau_{k}$ 's and the corresponding $a_{k}$ 's by applying 19 ; then, these rays are subtracted from the data in accordance with the structure of Eq. 10. Hence, the algorithm proceeds with the estimation of the lower rays, repeating the above process with the residual data, and so on until extracting the lowest ray (ray $\# K$ ).

Finally, the left-hand side of Eq. 20 is realized by applying the inverse fast Fourier transform (IFFT) to the 
frequency samples of $H\left(j \omega ; t_{n}\right) \cdot W_{B}(j \omega)$ with enough zeropadded values to represent continuous-time signals by means of discrete-time signals (sequences). The resulting estimation of path delays is very good and robust under noisy data; in fact, we have obtained good results in our simulations for signal-to-noise ratio below zero decibels. See Sect. 4 about simulation results for the details.

\subsection{First estimation of path coefficients}

Once the delays $\tau_{k}(k=1,2, \ldots, K)$ have been estimated, where $K$ is the maximum number of detected $\tau_{k}$ 's, the corresponding coefficients $a_{k}(k=1,2, \ldots, K)$ can be computed from Eq. 19. Note that in fact, we have $\tau_{k}\left(t_{n}\right)$ and $a_{k}\left(t_{n}\right)$ at time instant $t_{n}$, and we have to repeat the estimations for all time instants $t_{n}, n=1,2, \ldots, N$.

Finally, a precise estimation of vectors $\mathbf{a}$ and $\tau$ for a fixed $t_{n}$ can be done if we apply an optimization gradient algorithm after the first estimation given above. The details are given below.

\subsection{Refining estimations of path-delays and path-coefficients}

Suppose we have an initial approximation $\tau^{(0)}$ to $\tau$ that corresponds to a minimum in Eq. 12; from Eqs. 16 or 19, we compute an initial approximation $\mathbf{a}^{(0)}$ of $\mathbf{a}$ that corresponds to the minimum of Eq. 12, i.e.

$\mathbf{a}^{(0)}=\left(\mathbf{M}_{e}^{*(0)} \cdot \mathbf{M}_{e}^{(0)}\right)^{-1} \cdot \mathbf{M}_{e}^{*(0)} \cdot \mathbf{h}$,

where matrix $\mathbf{M}_{e}^{(0)}$ is computed from Eq. 18 for $\tau=\tau^{(0)}$

For $i=1,2, \ldots, I$ compute $\tau^{(i)}$ from $\tau^{(i-1)}$ and $\mathbf{a}^{(i-1)}$ according to the gradient algorithm:

$\tau^{(i)}=\tau^{(i-1)}-\mu \cdot \nabla_{\tau}^{(i-1)}(\varepsilon(\mathbf{a}, \tau))$

and $\mathbf{a}^{(i)}$ in accordance with Eq. 19, as follows

$\mathbf{a}^{(i)}=\left(\mathbf{M}_{e}^{*(i)} \cdot \mathbf{M}_{e}^{(i)}\right)^{-1} \cdot \mathbf{M}_{e}^{*(i)} \cdot \mathbf{h}$

where $\tau^{(i)}$ is the value of $\tau$ for the iteration $i, \mu$ is the step parameter that controls the convergence speed and the stability of the algorithm, and column vector $\nabla_{\tau}^{(i)}(\varepsilon(\mathbf{a}, \tau))$ is the gradient of $\varepsilon(\mathbf{a}, \tau)$ with respect to vector $\tau$ for $\mathbf{a}=\mathbf{a}^{(i)}$ and $\boldsymbol{\tau}=\boldsymbol{\tau}^{(i)}$, i.e. $\nabla_{\tau}^{(i)}(\varepsilon(\mathbf{a}, \boldsymbol{\tau}))=\left[\nabla_{\tau}(\varepsilon(\mathbf{a}, \boldsymbol{\tau}))\right]_{\mathbf{a}=\mathbf{a}^{(i)}, \boldsymbol{\tau}=\boldsymbol{\tau}^{(i)}}$ and, after some manipulations,

$$
\begin{aligned}
\nabla_{\tau}(\varepsilon(\mathbf{a}, \tau)) & =2 \mathrm{R}_{e}\left\{\sum_{m=1}^{M}\left(\frac{\partial e_{m}(\mathbf{a}, \tau)}{\partial \tau}\right)^{*} \cdot e_{m}(\mathbf{a}, \tau)\right\} \\
& =2 \mathrm{I}_{m}\left\{\left(\mathbf{M}_{e} \cdot \operatorname{diag}(\mathbf{a})\right)^{*} \cdot \operatorname{diag}(\boldsymbol{\omega}) \cdot\left(\mathbf{h}-\mathbf{M}_{e} \cdot \mathbf{a}\right)\right\}
\end{aligned}
$$

and matrix $\mathbf{M}_{e}^{(i)}$ is computed from Eq. 18 for $\tau=\tau^{(i)}$. The other parameters are given above from Eqs. 16 to 18. The stopping value $I$ (last iteration) is considered when $\tau^{(I)}$ is close enough to $\tau^{(I-1)}$ (for example, by considering a relative difference less than 5\%). Also, note that Eq. 17 can be obtained from formula Eq. 24 equaled to zero.

Finally, it is important to note that the estimation refinement is possible if the measured data are not too noisy (e.g. from our simulations: signal-to-noise ratio greater than $10 \mathrm{~dB}$ ); otherwise, the first estimation of path delays given above in Sect. 3.1 can not be improved by the refining Eq. 22. Our algorithm realizes a test to accept or reject the computed refinements according to the computed errors Eq. 12 for estimated values $\boldsymbol{\tau}=\boldsymbol{\tau}^{(i)}$ and $\mathbf{a}=\mathbf{a}^{(i)}$. Anyway, the first estimations $\tau^{(0)}$ and $\mathbf{a}^{(0)}$ given in Sects. 3.2 and 3.3, respectively, are fairly good for noisy data.

To summarize the estimation process, the first pathdelay estimations $\tau$ are realized by a peak detector over the band-limited impulse response shown in Eq. 20 of Sect. 3.1 ; then, the first path-coefficient estimations a are computed from Eq. 19. It is important to emphasize that low intensity rays (paths) are detected by the algorithm after removing the strongest rays from the data. So, the number of significant paths $K$ can be determined in an easy way and, then, the initial approximations $\tau^{(0)}$ and $\mathbf{a}^{(0)}$ are obtained. Refined estimations of $\tau$ and $\mathbf{a}$ from the initial approximations $\tau^{(0)}$ and $\mathbf{a}^{(0)}$ are shown in Sect. 3.3; these refined estimations are based on an iterative " $\tau$ - gradient and $\mathbf{a}$ - computation" algorithm, controlled by an error test and a stopping rule.

After the path delays $\tau_{k}\left(t_{n}\right)$ and the path coefficients $a_{k}\left(t_{n}\right)$ for $k=1,2, \ldots, K$, have been estimated at time instant $t_{n}$, we have to repeat the estimations for every time instants $t_{n}, n=1,2, \ldots, N$. This fact gives the possibility of applying the algorithm to non-stationary signals. Also, we can plot $\tau_{k}\left(t_{n}\right)$ versus $t_{n}$ for each $k$, realize histograms of $\tau_{k}$ (if it does not depend on $t_{n}$ ) or obtain statistical characteristics of $\tau_{k}\left(t_{n}\right)$. As for $a_{k}\left(t_{n}\right)$, we can plot the magnitude and phase versus $t_{n}$ for each $k$, obtain the spectral characteristics (e.g. power spectrum) of $a_{k}\left(t_{n}\right), n=1,2, \ldots, N$ for each $k$, etc. We will see these possibilities in the next sections.

The algorithm described above has been applied to a real ionospheric channel in the High Frequency (HF) band (carrier frequency of $14.8 \mathrm{MHz}$ ). The number of frequency samples is $M=73$ in an equivalent baseband channel of bandwidth $2,700 \mathrm{~Hz}$ approximately. The separation between two consecutive frequency samples is $37.5 \mathrm{~Hz}$. The number of time samples is $N=5,400$ taken with a sampling rate of $33 \mathrm{~ms}$. The observation time is about $3 \mathrm{~min}$. It is important to note that with a bandwidth of $2,700 \mathrm{~Hz}$, it is only possible to have a delay resolution about $0.2 \mathrm{~ms}$ in close rays with identical strengths. 


\section{Simulation results}

In order to test the proposed algorithm of Sect. 3 under simulated models, two cases have been studied. The first case corresponds to a deterministic multipath channel with additive noise and the second case corresponds to a random multipath channel with additive noise. The channel is the equivalent low-pass time-variant system $H(j \omega ; t)$, sampled at a rate $T=33 \mathrm{~ms}\left(t_{n}=n T, n=1,2, \ldots, N\right)$ and discrete frequencies: $\omega_{m}=2 \pi f_{m}$ with $f_{m}=f_{m-1}+37.5, m=1,2$, $\ldots, 73$ and $f_{0}=-37.5 \cdot(73-1) / 2=-1,350 \mathrm{~Hz}$, over a bandwidth of $2,700 \mathrm{~Hz}$ approximately. The problem to solve in this section consists on the estimation of the channel parameters from simulated data.

\subsection{Simulated deterministic channel}

As a first case, consider a deterministic multipath channel with additive noise, and try to estimate the channel parameters. Suppose, we have an $M \times N$ matrix $Y$ of complex noisy data, modeled as follows

$$
\begin{gathered}
Y(m, n)=\sum_{k=1}^{K} a_{k}(n) e^{-j \omega_{m} \cdot \tau_{k}(n)}+\eta(m, n) ; \\
m=1,2, \ldots, M ; n=1,2, \ldots, N,
\end{gathered}
$$

where $\eta(m, n)$ is a complex random sequence of white Gaussian noise of unit power per component (or power 2 for $\eta(m, n))$.

Our algorithm works for any values of $K, M$ and $N$. Different sets of parameter values have been used for testing the algorithms. However, for an easy graphical presentation, we use $K=3, M=73$ and $N=100$ in our simulation. Now, in order to show the algorithm resolution for delays $\tau_{k}$, we take the following path delays (in seconds): $\quad \tau_{1}(n)=0, \tau_{2}(n)=5 \cdot 10^{-4}, \tau_{3}(n)=2 n \cdot 10^{-5}+$ $10^{-3}$ for $1 \leq n \leq 50$, and $\tau_{3}(n)=1.5 \cdot 10^{-3}$ for $50<n \leq 100$, represented in Fig. 1 as dotted lines.

Also, in order to show the algorithm capacity for detecting paths, we consider that the path coefficients are $a_{1}(n)=4 \cdot e^{j \theta_{1}(n)}, a_{2}(n)=1 \cdot e^{j \theta_{2}(n)}$, and $a_{3}(n)=[2.5$ $\left.-\cos \left(3 \pi \cdot n \cdot 10^{-2}\right)\right] \cdot e^{j \theta_{3}(n)}$, being $\quad \theta_{1}(n)=-\theta_{2}(n)=$ $\frac{\pi}{6} \operatorname{sign}(\cos (3 \pi \cdot n / 100))+\frac{\pi}{3}$, and $\theta_{3}(n)=0$. The magnitude and phase representation of the coefficients can be seen in Fig. 2(a) and (b), respectively, as dotted lines. Note that the input signal-to-noise ratio (SNR) for coefficient $a_{k}(n)$ is defined as $\operatorname{SNR}\left(a_{k}\right)=\left|a_{k}\right|^{2} / 2$, i.e. $\operatorname{SNR}\left(a_{1}\right)=9 \mathrm{~dB}$, $S N R\left(a_{2}\right)=-3 \mathrm{~dB}$, and for coefficient $a_{3}(n): 0 \mathrm{~dB}<S N R$ $\left(a_{3}\right)<8 \mathrm{~dB}$.

In Figs. 1 and 2, we show the estimations of $\tau_{k}(n)$ and $a_{k}(n)$ for $k=1,2$ and 3, as continuous lines, from the knowledge of $Y(n, m)$ obtained in accordance with model

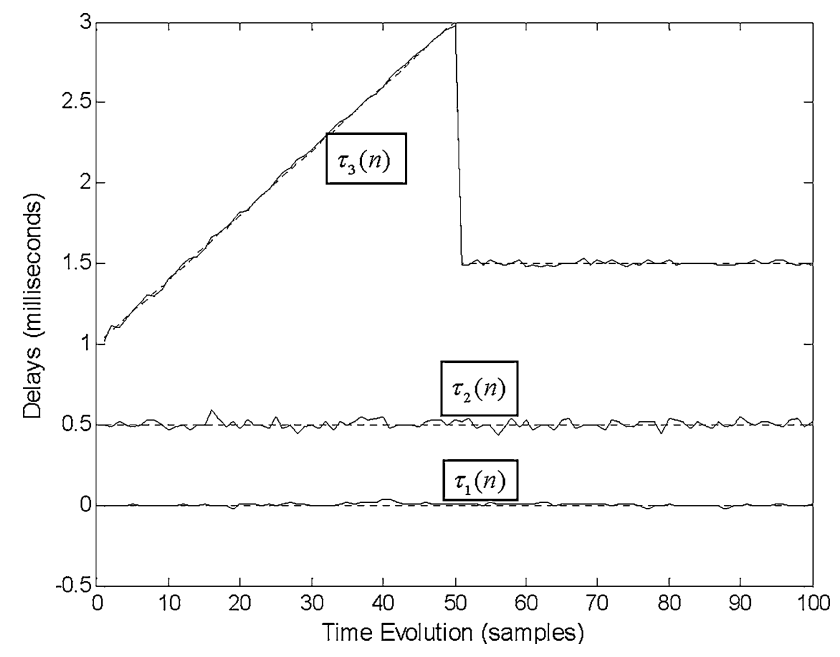

Fig. 1 Path delays versus time evolution (sample number) for the three paths of the deterministic model case: $\tau_{k}(n), k=1,2,3$ and $1 \leq n \leq 100$. Estimations (continuous lines) and exact values (dotted lines). The theoretical parameters of the channel are given in Sect. 4.1

Eq. 25. Figure 1 shows the three noisy lines of $\tau_{k}(n)$, $k=1,2$ and 3, where most absolute errors from the corresponding exact values are less than $0.05 \mathrm{~ms}$. Figure 2(a) shows the three noisy curves of the $a_{k}(n)$-magnitude: $\left|a_{k}(n)\right|, k=1,2$ and 3, where most absolute errors from the corresponding exact values are less than 0.25 (the same value for all curves). Figure 2(b) shows the three noisy lines of the $a_{k}(n)$-phase angle (in degrees): $\theta_{k}(n), k=1,2$ and 3 , where most absolute errors from the corresponding exact values are less than 3 degrees for $\theta_{1}(n)$, less than 5 degrees for $\theta_{3}(n)$, and less than 10 degrees for $\theta_{2}(n)$, i.e. phase errors are inversely related to the coefficient magnitudes. Also, although it is not shown here, if the coefficients $a_{k}(n)(k=1,2$ and 3$)$ of the model Eq. 25 are multiplied by 2 , corresponding to an increasing of $6 \mathrm{~dB}$ in the input SNR, the coefficient estimation errors are divided by 2 , but the delay estimations only improve slightly. Finally, a post-estimation smoothing can be applied in order to reduce more the noise in the delay and coefficient curves.

Now, some comments about the selection of the parameters should be provided. The estimation algorithm of path delays and coefficients works efficiently for any number of paths, and delays between 0 and $30 \mathrm{~ms}$ with a delay resolution about $0.2 \mathrm{~ms}$ (a half of the inverse of the transmission bandwidth, approximately). The signal level corresponding to each path can be below the noise (SNR $=-3 \mathrm{~dB})$, as is the case of path $k=2$, in the simulation presented above. Note that if at any time instant $n_{0}$ it occurs $\tau_{1}\left(n_{0}\right)=\tau_{2}\left(n_{0}\right)=\tau_{3}\left(n_{0}\right)$, then the algorithm considers only one delay $\tau_{1}\left(n_{0}\right)$ and only one coefficient $a_{1}\left(n_{0}\right)$ corresponding to the sum of the other tree 

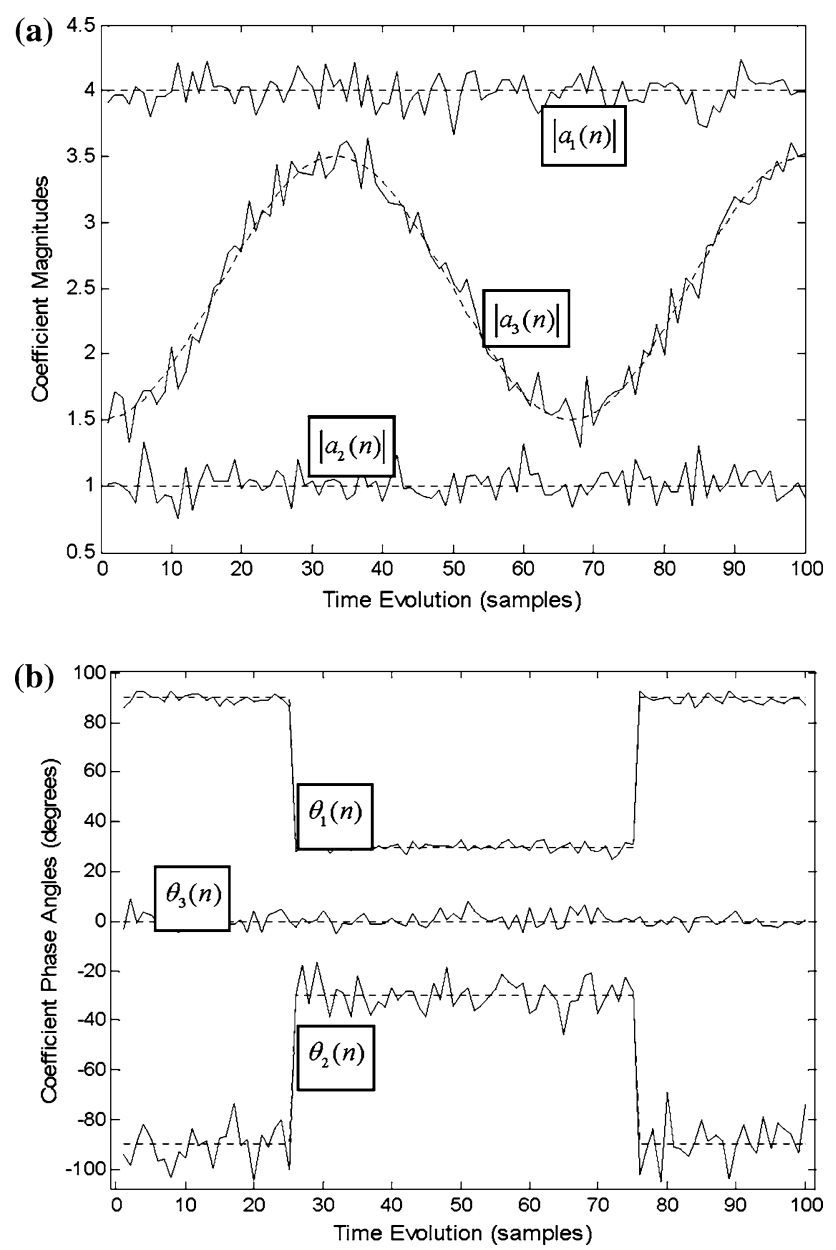

Fig. 2 Path coefficients versus time evolution (sample number) for the three paths of the deterministic model case: a Path-coefficient magnitudes: $\left|a_{k}(n)\right|, k=1,2,3$ and $1 \leq n \leq 100$, and $\mathbf{b}$ Coefficient phases: $\theta_{k}(n)=\operatorname{angle}\left[a_{k}(n)\right], k=1,2,3$. Estimations (continuous lines) and exact values (dotted lines). The theoretical parameters of the channel are given in Sect. 4.1

coefficients. So, we do not allow this possibility or any other ambiguity in our deterministic model in order to facilitate curves' presentation.

As it can be seen from Figs. 1 and 2, estimations of $\tau_{k}(n)$ and $a_{k}(n)$ are very good for the first and third paths (see above for values), but not so good for the second path (ray) for two reasons: the first ray masks (hides) the second one, and the second ray has a level below the noise. Finally, it is worth noting that our algorithm first estimates the most powerful rays and, then, theses rays are removed in order to estimate the less powerful and hidden rays, and so on, until the remaining ray peaks are of the same level of the noise peaks. This approach improves considerably the delay-resolution estimation. So, in our deterministic case, once the first and third rays are removed, the second ray is detected and, then, $\tau_{2}(n)$ and $a_{2}(n)$ are estimated.

\subsection{Simulated random channel}

Now, consider that the model shown before in Eq. 25 has random coefficients and constant delays, plus a random sequence of white Gaussian noise. We suppose the path delay $\tau_{k}$ is constant for each path $k$, and coefficients $a_{k}(n)$, $k=1,2, \ldots, K$, are complex Gaussian stationary random sequences of zero mean, and Gaussian autocorrelation function (or Gaussian power spectral density) given by

$$
\begin{aligned}
r_{k}(n) & =E\left\{a_{k}^{*}(l) \cdot a_{k}(l+n)\right\}=P_{k} \cdot \mathrm{e}^{-2\left(n T \cdot \pi \sigma_{k}\right)^{2}}, \\
k & =1,2, \ldots, K,
\end{aligned}
$$

where $P_{k}$ is the power of coefficient $a_{k}(n), T=0.033 \mathrm{~s}$ is the sampling period, and $\sigma_{k}$ is the coefficient Doppler spread. Also, it is supposed that coefficients are mutually independent. Then, the model is similar to that in Eq. 25, but all $\tau_{k}$ 's do not depend on $n$, and $a_{k}(n)$ are random sequences.

For the simulation, we set $K=3, N=50,000$ samples (or an observation time interval of $27.5 \mathrm{~min}$ ). The path delays are: $\tau_{1}=0, \tau_{2}=0.5$ and $\tau_{3}=1.5 \mathrm{~ms}$. The coefficient powers are: $P_{1}=16, P_{2}=1$ and $P_{3}=4$, and the noise power is $E\left\{\eta^{*}(m, n) \cdot \eta(m, n)\right\}=2$; so the signal-tonoise ratio (SNR) for each coefficient is $\operatorname{SNR}\left(a_{k}\right)=P_{k} / 2$ i.e. $\operatorname{SNR}\left(a_{1}\right)=9 \mathrm{~dB}, \operatorname{SNR}\left(a_{2}\right)=-3 \mathrm{~dB}$ and $\operatorname{SNR}\left(a_{3}\right)=$ $3 \mathrm{~dB}$. Also, we consider $\sigma_{k}=0.1 \mathrm{~Hz}$ as channel Doppler spread in Eq. 26 for $k=1,2$ and 3.

We try to estimate the path delays $\tau_{k}$ for $k=1,2$ and 3, from the delay histogram. Also, we estimate the power spectrum of the path coefficients by, first, estimating the coefficients $a_{k}(n)$ for $k=1,2$ and 3, and for $n=1,2, \ldots$, $N$; and, then, realizing a spectrum estimation for each coefficient. The power spectrum estimation is based on Welch's method by averaging the periodogram over subsequences of noisy coefficient estimations [9, pp. 1163].

In Fig. 3 we show histograms of the delay estimations, where we can clearly see the three peaks (modes) of the corresponding ray delays. The final delay estimations are the medians $\left(\hat{\tau}_{k}\right)$ over the corresponding intervals, each one containing only one peak, and resulting in $\hat{\tau}_{1}=6.5$. $10^{-6}, \hat{\tau}_{2}=5.1 \cdot 10^{-4}$ and $\hat{\tau}_{3}=1.51 \cdot 10^{-3} \mathrm{~s}$. According to our simulation results, it is required at least $\operatorname{SNR}\left(a_{k}\right)=$ $-12 \mathrm{~dB}$ to detect the corresponding $k t h$-ray with a high reliability by means of our estimation algorithm. The reason of a so low SNR, required for detecting the corresponding ray, is based on the histogram estimation: for very low SNR, the corresponding histogram is very wide but it does not lose the bell-shaped aspect, and the mode value is well defined (i.e. $\hat{\tau}_{k}$ is easily computed).

In Fig. 4, it can be seen the power spectral density of the coefficients, where we have used a sequence of 50,000 samples: 100 sections with 500 samples each for the power 


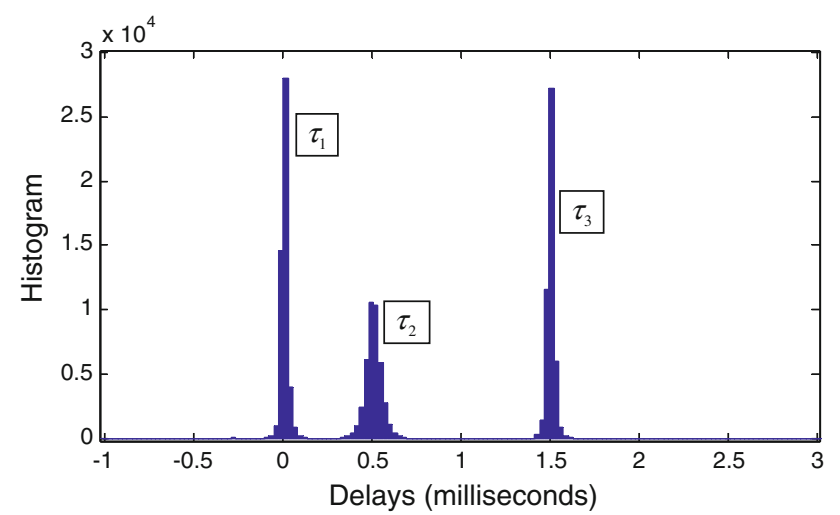

Fig. 3 Delay histograms of path-delay estimations for a three-path channel with random path coefficients $(50,000$ samples of the delay sequences). The theoretical parameters of the channel are given in Sect. 4.2

spectrum estimation; also, it is shown the exact power spectral density for comparisons. Finally, if a more estimation improvement is required, we have to increase the sequence length for a better estimation resolution, and to increase the number of sequence sections for a better precision.

\section{Experimental results: a real ionospheric channel}

Finally, we consider a real (actual) ionospheric High Frequency (HF) narrowband channel, and we try to estimate the channel parameters from measured data. The measurement system is a sounder based on an Orthogonal Frequency-Division Multiplexing (OFDM) system, where we are able to estimate a set of samples of the frequency response over time.

\subsection{Sounder description}

The sounder we have used is an OFDM system where all transmitted symbols are pilots. Although there are other

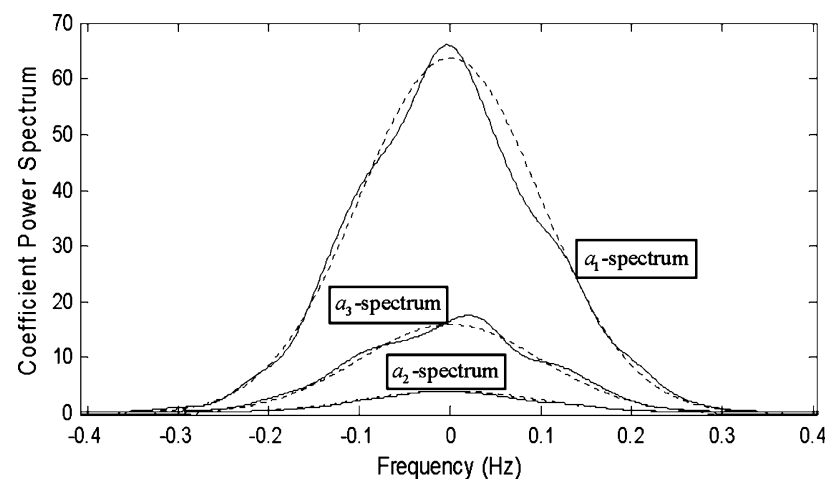

Fig. 4 Power spectral density of each coefficient for a three-path channel with random coefficients: estimation (continuous lines) and exact value (dotted lines). The theoretical parameters of the channel are given in Sect. 4.2 waveforms for this purpose, probably even more robust, we have utilized the OFDM technique because we had already designed and tested several modems for digital voice and data transmission using this technique. Considering Software Defined Radio (SDR) principles, the complexity of this new design has been the minimum one, just considering that all transmitted symbols are known. In Figs. 5(a) and (b), it is shown block diagrams of the transmitter and the receiver sides, respectively. Let us make some comments on some specific features of this approach.

We point out that we require an accurate time and frequency synchronization at the receiver side, in order to guarantee the orthogonality property among the sub-carriers, useful for the appropriate estimation of the frequency response. The frequency correction block cancels the frequency offset between transmitter and receiver local oscillators, and it also cancels the mean Doppler shift of the channel. So, analyzing a long probe signal, we can estimate the Doppler spread of the channel. The synchronization algorithm is strongly based on [12] where a full symbol as a header of the transmission is included with a particular feature, i.e. a time replicated structure that allows the robust frequency and time estimation.

One terminal is in Madrid and the other is $1,800 \mathrm{~km}$ away in Las Palmas de Gran Canaria (The Canary Islands).

(a)

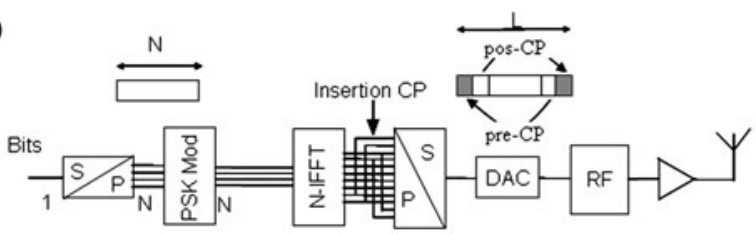

(b)

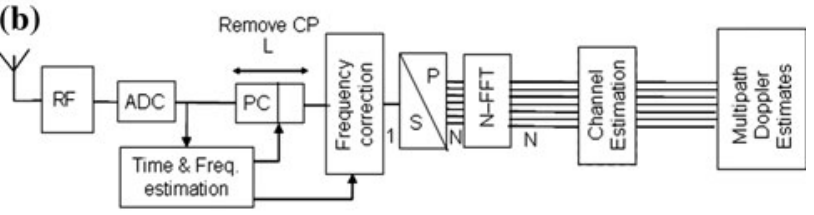

(c)
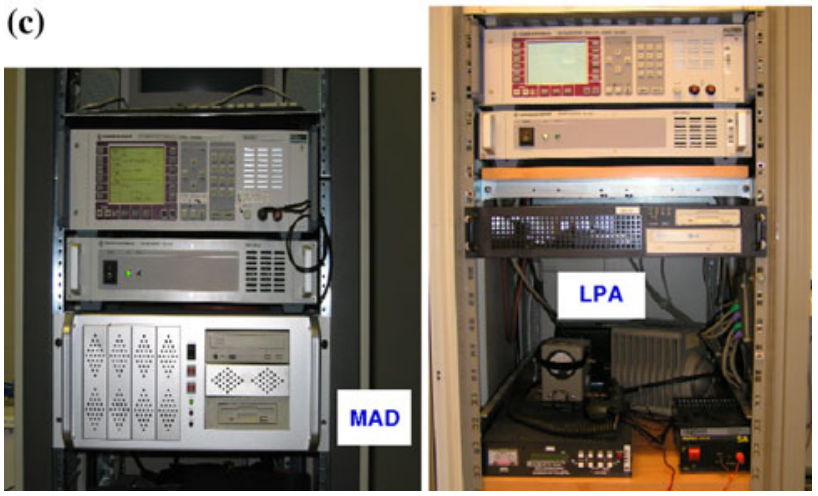

Fig. 5 a Transmitter side of the Sounder Block-Diagram. b Receiver side of the Sounder Block-Diagram. c HF measurement system: platform of Madrid (MAD) and platform of Las Palmas (LPA) 
Photographs of both platforms are given in Fig. 5(c). Suppose, we have a $M \times N$ matrix: $Y\left(\omega_{m}, t_{n}\right) \approx$ $H\left(j \omega_{m} ; t_{n}\right), m=1,2, \ldots, M ; n=1,2, \ldots, N$ of complex data (complex envelope), measured by the HF modem at a rate of $9,600 \mathrm{~b} / \mathrm{s}$ with a carrier frequency of $14.8 \mathrm{MHz}$ and a bandwidth of $2,737 \mathrm{~Hz}$. The Cyclic Prefix (CP) is $3.3 \mathrm{~ms}$ (Pre-CP and Post-CP). The multipath model is given in Eq. 10. Our purpose is to estimate the number $K$ of ionospheric rays, the complex amplitudes $a_{k}(t)$ and the delays $\tau_{k}(t)(k=1,2, \ldots, K)$ from Eq. 10, considering the following parameter values: $t_{n}=n T(n=1,2, \ldots, N)$ with $T=1 / 30 \approx 0.033 \mathrm{~s}$ and $N=5,450$ time samples (or an observation time of $3 \mathrm{~min}$, approximately), and $\omega_{m}=$ $2 \pi f_{m}$, with $f_{m}=f_{m-1}+37.5, m=1,2, \ldots, M$, and $M=73$ frequency samples.

\subsection{Experimental results}

The matrix of data $Y\left(\omega_{m}, t_{n}\right) \approx H\left(j \omega_{m} ; t_{n}\right), m=1,2$, $\ldots, M ; n=1,2, \ldots, N$ was measured repeatedly 12 times in one day $(24 \mathrm{~h})$ and here we show the most important results. The ionospheric link was established between Madrid and the Canary Islands on the first of April, 2008. Firstly, we have to say that at night (say, from local time 22:00 to local time 6:00, approximately) no link was possible (no path or ray has been detected). In the sun times, some paths or rays are possible: a strong ray and others slighter. In Figs. 6 and 7, we show results of one link (at local time 14:00, approximately, for $3 \mathrm{~min}$ ). Other alternative results for links at different times can be seen in [13] (link at local time 06:00 and link at local time 12:00, approximately, for $3 \mathrm{~min}$ ).

Specifically, Fig. 6(a) shows delay realization sections of three possible paths, where estimation (median) delays are $\hat{\tau}_{1}=0.021, \hat{\tau}_{2}=0.47$ and $\hat{\tau}_{3}=-0.25 \mathrm{~ms}$ (the strongest ray indicates the reference delay, i.e. $\hat{\tau}_{1} \approx 0$ ). Note that ray \#3 arrives first, then ray \#1 (the strongest ray) and, then, ray \#2. Note that there are some temporal variations (deterministic and random) in delay estimations $\tau_{k}(n T)$, being different for each $k$ and, also, different for each time of the day; eventually, these delay variations are related to the motion of the ionospheric layers. Also, Fig. 6(b) shows delay histograms for all possible path estimations, and we can see up to four paths defined by the corresponding delays, although path number four has a signal level too low or much below the noise level (according to the histogram, the number of detected cases is about $15 \%)$. The temporal variations in delay estimations $\tau_{k}(n T)$, appearing in Fig. 6(a), have little influence in the histograms of Fig. 6(b); consequently, these variations cause slight modifications on the delay estimations (median values).
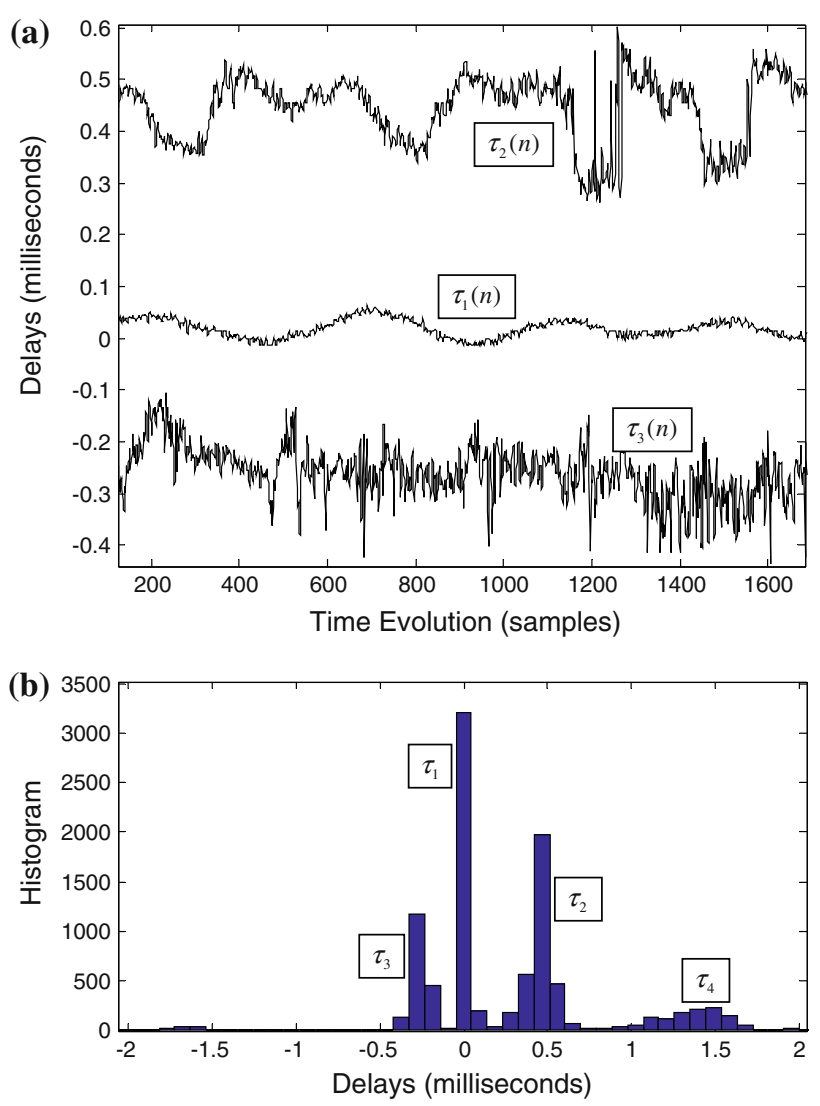

Fig. 6 Path-delay estimations for a real (actual) ionospheric channel. a Path-delay estimations versus time evolution (sample number) for the three strongest paths: $\tau_{k}(n), k=1,2,3$ and $200<n<1600$ (The temporal interval between two consecutive samples is $T=33 \mathrm{~ms}$ ). (b) Delay Histograms for 3,400 samples of the delay sequences. The measurement conditions are given in Sect. 5

Finally, Fig. 7(a) shows coefficient-magnitude realizations of the three strongest paths (rays), where the strongest ray is about 4-times the second strongest one and 10-times the third one. The estimated signal-to-noise ratio (SNR) for each coefficient is approximately as follows: $\operatorname{SNR}\left(a_{1}\right)=$ $16 \mathrm{~dB}, \operatorname{SNR}\left(a_{2}\right)=3 \mathrm{~dB} . \operatorname{SNR}\left(a_{3}\right)=-5 \mathrm{~dB}$ and $\operatorname{SNR}\left(a_{4}\right)$ $=-8 \mathrm{~dB}$. In Fig. 7(b), we show estimations of power spectral densities, where we have used a sequence with 5,400 samples (20 sections of 270 samples each one) for each coefficient. All curves are bell-shaped with approximately zero-mean Doppler frequency and a Doppler-frequency spread close to $0.1 \mathrm{~Hz}$. Because the modem receiver tunes automatically the mean received frequency, it is not possible to estimate the mean Doppler frequency of the path coefficients. Finally, according to our measured results, the total power is about 12.1 units, and the powers of the four rays are approximately: $P_{1}=11, P_{2}=$ $0.6, P_{3}=0.1$ and $P_{4}=0.05$; if we remove all the ray signals from the received signal Eq. 10, it remains a flat power spectrum that corresponds to noise and interference with approximately a power of 0.3 units. 

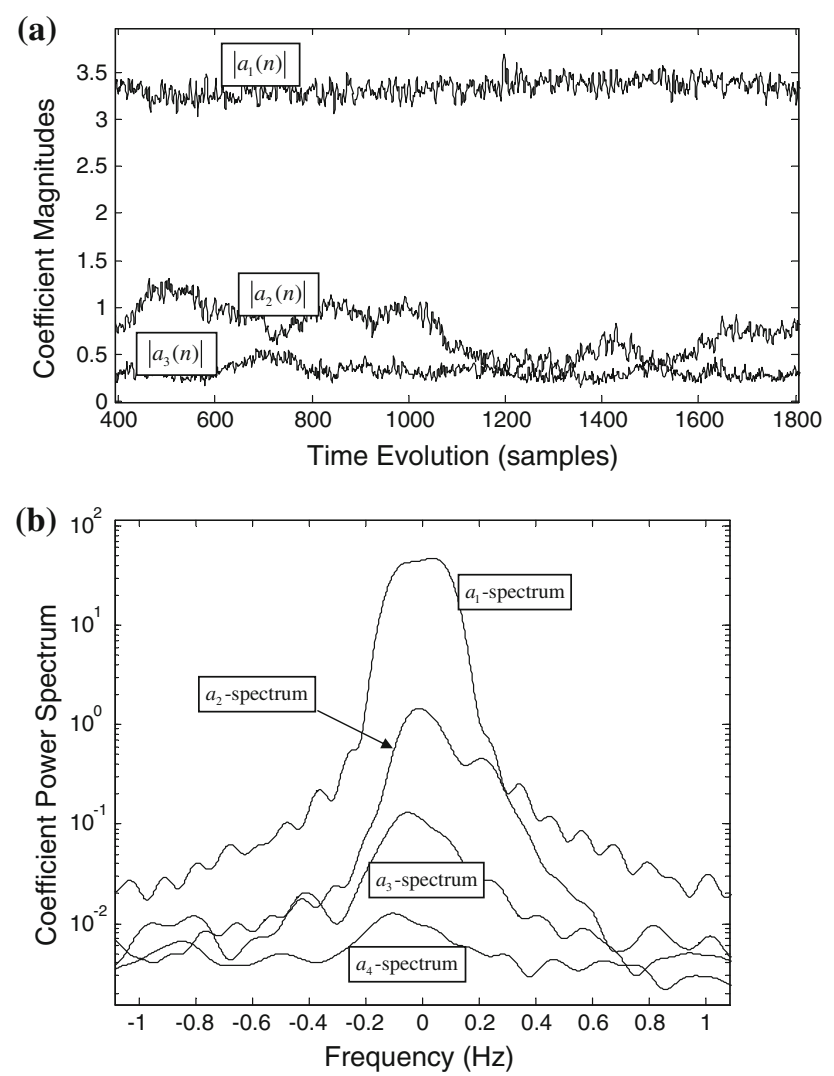

Fig. 7 Random coefficient realizations and coefficient power spectrum estimations for a real (actual) ionospheric channel. a Coefficient-magnitude estimations versus time evolution (sample number) for the three strongest paths: $\left|a_{k}(n)\right|, k=1,2,3$ and $400 \leq n \leq 1800$ (The temporal interval between two consecutive samples is $T=33 \mathrm{~ms}$ ). b Power Spectral Density of each coefficient for the four strongest paths: $a_{k}-$ spectrum. The measurement conditions are given in Sect. 5

\section{Conclusions}

In this paper, the theory of linear time-variant systems has been applied to multipath channels and, in particular, to ionospheric channels. We have used the concepts of impulse and frequency responses of linear time-variant systems, and provided some useful formulas in the frequency domain for both deterministic and random channels, derived from the two-dimensional Fourier Transform of the impulse response.

Also, we have designed and implemented algorithms for estimating path delays and path coefficients. These algorithms have been tested exhaustively over deterministic and random multipath channel models. The results have shown very good path-delay estimations and good pathcoefficient estimations. Also, the algorithms have shown high ability for detecting low intensity paths.

Our algorithms have been applied to parameter estimations of real (actual) HF ionospheric channels, showing high capability for ray detection, and performing precise estimations (including temporal variations) of ray delays. Finally, the power spectral density has been estimated for each ray coefficient, resulting in bell-shaped curves with a mean Doppler frequency close to zero and a Doppler-frequency spread close to $0.1 \mathrm{~Hz}$.

Finally, the analysis (carried out from real data) shows temporal variations of the path coefficients and path delays. Therefore, further research work is required to characterize this time variability, in order to provide a satisfactory Doppler spectrum model of the path coefficients, related to the temporal variation of each path-delay sequence (corresponding to each ionospheric layer motion). Also, this improved model will probably provide a better predictability of the ionospheric propagation, and a better understanding of the suitability of different transmitting waveforms.

Acknowledgments This work has been partially supported by the "Dirección General de Investigación" of the "Ministerio de Educación y Ciencia" under Grant TEC2007-67520-C02/TCM, the "Universidad Politécnica de Madrid" and the "Universidad de Las Palmas de Gran Canaria”, Spain.

The authors are very grateful to the anonymous reviewers for their useful suggestions.

\section{Appendix 1}

Frequency response of linear time-variant systems

The convolution integral Eq. 1 of a linear time-variant system can be expressed in equivalent ways as follows

$y(t)=\int_{-\infty}^{\infty} h(\tau ; t) x(t-\tau) d \tau=\int_{-\infty}^{\infty} h(t-\tau ; t) x(\tau) d \tau$.

If $X(j \omega)$ is the Fourier transform of $x(t)$, we can write from Eq. 27

$$
\begin{aligned}
y(t) & =\int_{-\infty}^{\infty} h(\tau ; t)\left[\frac{1}{2 \pi} \int_{-\infty}^{\infty} X(j \omega) e^{j \omega(t-\tau)} d \omega\right] d \tau \\
& =\frac{1}{2 \pi} \int_{-\infty}^{\infty} X(j \omega) H(j \omega ; t) e^{j \omega t} d \omega,
\end{aligned}
$$

where $H(j \omega ; t)$ is the system function or the Fourier transform of $h(\tau ; t)$ with respect to the first variable $\tau$ (delay variable), i.e.

$H(j \omega ; t)=\int_{-\infty}^{\infty} h(\tau ; t) e^{-j \omega \tau} d \tau$

Formula Eq. 28 is important and has a similar interpretation in linear time-invariant systems, where 
$H(j \omega ; t)$ does not depend on time $t$. As an example, it is illustrative to consider the input signal $x(t)=e^{j \omega_{0} t}$, then from Eqs. 27 or 28 , we have $y(t)=H\left(j \omega_{0} ; t\right) \cdot e^{j \omega_{0} t}$ that can be interpreted as an amplitude modulation of the input signal $e^{j \omega_{0} t}$ by the system function $H\left(j \omega_{0} ; t\right)$ or, in the frequency domain, the input spectral line $2 \pi \delta\left(\omega-\omega_{0}\right)$ is spread out at the output according to the spectrum of $H\left(j \omega_{0} ; t\right)$.

Also, formula Eq. 28 can be expressed in the frequency domain, as follows

$$
\begin{aligned}
Y(j \Omega) & =\frac{1}{2 \pi} \int_{-\infty}^{\infty} X(j \omega) \tilde{H}(j \omega ; j(\Omega-\omega)) d \omega \\
& =\frac{1}{2 \pi} \int_{-\infty}^{\infty} X(j(\Omega-\omega)) \tilde{H}(j(\Omega-\omega) ; j \omega) d \omega
\end{aligned}
$$

where $Y(j \Omega)$ and $\tilde{H}(j \omega ; j \Omega)$ are the Fourier transforms of $y(t)$ and $H(j \omega ; t)$, respectively, over time variable $t$. That is,

$$
\begin{aligned}
\tilde{H}(j \omega ; j \Omega) & =\int_{-\infty}^{\infty} H(j \omega ; t) e^{-j \Omega \cdot t} d t \\
& =\int_{-\infty}^{\infty} \int_{-\infty}^{\infty} h(\tau ; t) e^{-j(\omega \tau+\Omega t)} d \tau d t .
\end{aligned}
$$

Remarks Compare formulas Eqs. 30 and 27 representing convolution integrals in the frequency domain and in the time domain, respectively. Also, note that if $h(\tau ; t)=$ $h_{0}(\tau)$, i.e. the system is time-invariant, then from Eq. 31 $\tilde{H}(j \omega ; j \Omega)=H_{0}(j \omega) \cdot 2 \pi \delta(\Omega)$, and from Eq. 30 we have $Y(j \Omega)=X(j \Omega) \cdot H_{0}(j \Omega)$, being the well-known formula applied to linear time-invariant systems.

Finally, from Eqs. 2 and 31, we have

$$
\tilde{H}(j \omega ; j \Omega)=G(j \omega) \sum_{k=1}^{K} \int_{-\infty}^{\infty} a_{k}(t) e^{-j \omega \cdot \tau_{k}(t)} \cdot e^{-j \Omega \cdot t} d t
$$

Assuming that delay $\tau_{k}(t)$ can be approximated as $\tau_{k}(t) \approx \tau_{k}+v_{k} \cdot t$, then Eq. 32 could be expressed as

$\tilde{H}(j \omega ; j \Omega) \approx G(j \omega) \sum_{k=1}^{K} e^{-j \omega \cdot \tau_{k}} A_{k}\left(j\left(\Omega+\omega \cdot v_{k}\right)\right)$,

where $A_{k}(j \Omega)$ is the Fourier transform of $a_{k}(t)$, and from Eqs. 33 and 30 where symbol * means convolution operation.

Remarks Expression Eq. 34 has the following interpretation: for each path $k$, the path input-signal spectrum $G(j \Omega) X(j \Omega)$ undergoes a linear phase change due to the mean delay $\tau_{k}$, i.e. $G(j \Omega) X(j \Omega) e^{-j \Omega \tau_{k}}$; then, it undergoes a frequency expansion by a factor $1-v_{k}$; hence, the result is convolved by $A_{k}(j \Omega)$, resulting in a larger spectral spread of the input-signal spectrum. Finally, the output-signal spectrum is the sum of the output-signal spectra corresponding to all paths.

\section{Appendix 2}

Output power spectrum of linear time-variant systems

In the case of considering stationary random processes, we will develop the corresponding formulas for the power spectral density or the power spectrum of the output signal.

If $h(\tau ; t)$ and $x(t)$ are independent random processes and $x(t)$ is stationary, we can define the autocorrelation function $r_{y}(t, \Delta t)$ of $y(t)$ as follows

$$
\begin{aligned}
& r_{y}(t, \Delta t)=E\left\{y^{*}(t) \cdot y(t+\Delta t)\right\} \\
& =\int_{-\infty}^{\infty} \int_{-\infty}^{\infty} E\left\{h^{*}(u ; t) h(v ; t+\Delta t)\right\} \cdot r_{x}(\Delta t+u-v) d u d v
\end{aligned}
$$

where $y^{*}(t)$ is the complex conjugate of $y(t), r_{x}(\Delta t)=$ $E\left\{x^{*}(t) \cdot x(t+\Delta t)\right\}$ is the autocorrelation function of the stationary input $x(t)$ and $E\{z\}$ is the statistical expectation of $z$. Identifying $\tau=u$ and $\Delta \tau=v-u$, we can write

$r_{y}(t, \Delta t)=\int_{-\infty}^{\infty} \int_{-\infty}^{\infty} r_{h}(\tau, \Delta \tau ; t, \Delta t) \cdot r_{x}(\Delta t-\Delta \tau) d \tau d(\Delta \tau)$

where $r_{h}(\tau, \Delta \tau ; t, \Delta t)=E\left\{h^{*}(\tau ; t) h(\tau+\Delta \tau ; t+\Delta t)\right\}$ is the autocorrelation function of the impulse response $h(\tau ; t)$.

Finally, supposing $h(\tau ; t)$ is stationary in time $t$, then $y(t)$ is also stationary, and Eq. 36 can be written as

$$
\begin{aligned}
Y(j \Omega) & =\sum_{k=1}^{K} \frac{1}{2 \pi} \int_{-\infty}^{\infty} A_{k}\left(j\left(\Omega-\left(1-v_{k}\right) \omega\right)\right) G(j \omega) X(j \omega) e^{-j \omega \cdot \tau_{k}} d \omega \\
& =\frac{1}{2 \pi\left(1-v_{k}\right)} \sum_{k=1}^{K} A_{k}(j \Omega) *\left[G\left(j \Omega /\left(1-v_{k}\right)\right) X\left(j \Omega /\left(1-v_{k}\right)\right) e^{-j \Omega \cdot \tau_{k} /\left(1-v_{k}\right)}\right],
\end{aligned}
$$


$r_{y}(\Delta t)=\int_{-\infty}^{\infty} r_{h}(\Delta \tau ; \Delta t) \cdot r_{x}(\Delta t-\Delta \tau) d(\Delta \tau)$,

where

$$
\begin{aligned}
r_{h}(\Delta \tau ; \Delta t) & =\int_{-\infty}^{\infty} r_{h}(\tau, \Delta \tau ; t, \Delta t) d \tau \\
& =\int_{-\infty}^{\infty} E\left\{h^{*}(\tau ; t) h(\tau+\Delta \tau ; t+\Delta t)\right\} d \tau .
\end{aligned}
$$

Compare equation Eq. 37 referred to autocorrelation functions of signals with Eq. 1 referred to signals.

In the frequency domain, if $S_{y}(j \Omega)$ is the power spectral density of $y(t)$, i.e. the Fourier transform of $r_{y}(\Delta t)$, and $S_{x}(j \Omega)$ is the power spectral density of $x(t)$, then Eq. 37 is transformed into

$S_{y}(j \Omega)=\frac{1}{2 \pi} \int_{-\infty}^{\infty} S_{x}(j \omega) S_{h}(j \omega ; j(\Omega-\omega)) d \omega$,

where

$S_{h}(j \omega ; j \Omega)=\int_{-\infty}^{\infty} \int_{-\infty}^{\infty} r_{h}(\Delta \tau ; \Delta t) e^{-j(\omega \Delta \tau+\Omega \Delta t)} d(\Delta \tau) d(\Delta t)$.

Note the similarity between Eqs. 39 and 30, so a similar interpretation could be done here.

\section{References}

1. Zadeh, L. A. (1950). Frequency analysis of variable networks. Proceedings of the I.R.E, 38(3), 291-299.

2. Kailath, T. (1962). Measurements on time-variant communication channels. IRE Transactions on Information Theory, 8(5), 229-236.

3. Bello, P. A. (1963). Characterization of randomly time-variant linear channels. IEEE Transactions on Communications, 11(4), 360-393.

4. Bello, P. A. (2007). Evaluation of mobile ultra wideband modems in dense multipath-Part 1: Channel model. IEEE Transactions on Wireless Communications, 6(11), 4145-4153.

5. Watterson, C. C., Juroshek, J. R., \& Bensema, W. D. (1970). Experimental confirmation of an $\mathrm{HF}$ channel model. IEEE Transactions on Communications, 18(6), 792-803.

6. Leconte, M., \& Testard, M. (1997). A model of high frequencies (H.F) channel used to design a modem of 9600 bits/s rate in $3 \mathrm{kHz}$ of bandwidth. Proceedings of IEEE Military Communications Conference (MILCOM), 1, 351-355.

7. Mastrangelo, J. F., Lemmon, J. J., Vogler, L. E., Hoffmeyer, J. A., Pratt, L. E., \& Behm, C. J. (1997). A new wideband high frequency channel simulation system. IEEE Transactions on Communications, 45(1), 26-34.
8. Gherm, V. E., Zernov, N. N., \& Strangeways, H. J. (2003). Wideband HF simulator for multipath ionospherically reflected propagation channels. Proceedings of the 12th International Conference on Antennas and Propagation (ICAP 2003), 1, 128-131.

9. Mitra, S. K., \& Kaiser, J. F. (Eds.). (1993). Handbook for Digital Signal Processing. New York: Wiley.

10. Proakis, J. G. (1995). Digital Communications (3rd ed.). New York: McGraw-Hill, Inc.

11. Meyr, H., Moeneclaey, M., \& Fechtel, S. A. (1998). Digital Communication Receivers (Synchronization, Channel Estimation, and Signal Processing). New York: Wiley.

12. Schmidl, T. M., \& Cox, D. C. (1997). Robust frequency and timing synchronization for OFDM. IEEE Transactions on Communications, 45(12), 1613-1621.

13. Sanz-González, J. L., Zazo-Bello, S., Pérez-Álvarez, I. A., \& López-Pérez, J. (2009). Parameter estimation algorithms for ionospheric channels. Proceedings of the 11th IET International Conference on Ionospheric Radio Systems and Techniques (IRST'2009), 4-8.

\section{Author Biographies}

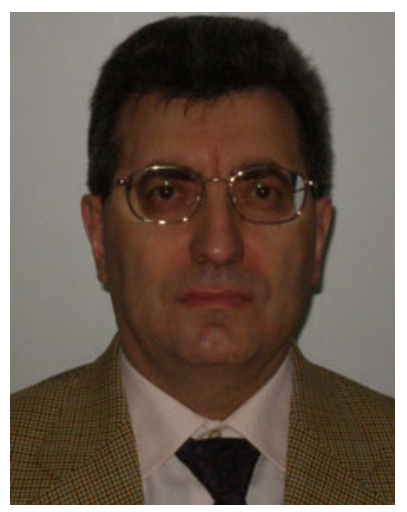

José L. Sanz-González received the Telecommunication Engineer and Doctor degrees from "Escuela Técnica Superior de Ingenieros de Telecomunicación" (ETSIT), Universidad Politécnica de Madrid (UPM), Spain. Dr. Sanz-González is a Full Professor of the Department "Señales, Sistemas y Radiocomunicaciones" at ETSIT-UPM. His area of scientific interest is deterministic and statistical signal processing, and he does research work on simulation and optimization of nonparametric detectors applied to radar and communication systems. Also, Prof. Sanz-González collaborates with the industry in development projects on digital transmission. He is coauthor of three textbooks and more than a hundred of reports and papers published in technical journals and conference proceedings.

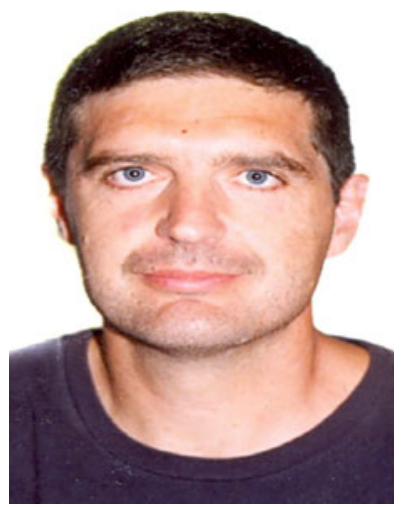

Santiago Zazo is Telecom Engineer and Dr. Engineer by the Universidad Politécnica de Madrid (UPM) in 1990 and 1995, respectively. From 1991 to 1994 he was with the University of Valladolid and from 1995 to 1997 with the University Alfonso X El Sabio in Madrid. In 1998 he joined UPM as Associate Professor in Signal Theory and Communications. His main research activities are in the field of Signal Processing with applications to Audio, Communications and Radar. More recently, he has been mostly focused on MIMO communications and Wireless Sensor Networks from both physical layer and networking points of view. Since 1990 he is author/coauthor of more than 10 journal papers and about 90 
conference papers. He has also led many private and national Spanish projects and has participated in five European projects, leading three of them. He is leading the HF communication group at UPM with large activity in publications and technological transfer to civil and military applications in this field.

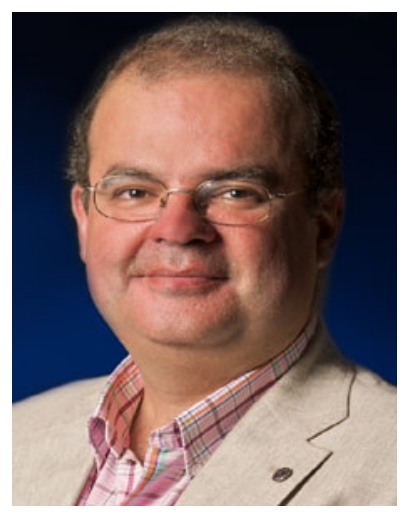

Ivan A. Pérez-Álvarez is Telecom Engineer and Dr. Engineer by the Universidad Politécnica de Madrid (Spain) in 1990 and 2000, respectively. From 1989 to 1997 he was in Europea de Comunicaciones S.A. and Telefónica Sistemas S.A., where he was a member staff of Special Projects Department and working in the Digital Signal Processing Group. He was involved in the design of $\mathrm{HF} / \mathrm{VHF} / \mathrm{UHF}$ digital communications systems for the Spanish Ministry of Defence. He joined the Universidad de Las Palmas de Gran Canaria (Spain) as an Associate Professor in 1998. At
2006 he was Head of Technological Centre for Innovations in Communications until 2010 and at the present is the vice-director of Institute for Technological Development and Innovation in Communications (IDeTIC). His main research activities are in the field of Signal Processing for Radio Communications.

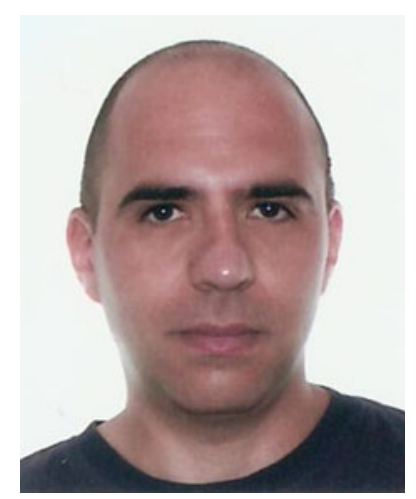

Javier López-Pérez received his MS Degree in Telecommunication Engineering from the University of Las Palmas de Gran Canaria (ULPGC), Spain, in 2004. He works as a Research Assistant for the Institute for Technological Development and Innovation in Communications (IDeTIC) of ULPGC, where he is working towards his $\mathrm{PhD}$ Degree in the fields of MultiCarrier Communications, Software Defined Radio and DSP Real Time Signal Processing. 